\title{
Neuro-based Olfactory Model for Estimation of Sensory Characteristic of Mice
}

\author{
Zu Soh*, Toshio Tusji*, Noboru Takiguchi ${ }^{\dagger}$ and Toshio Tsuji ${ }^{\ddagger}$ \\ * Graduate School of Engineering, Hiroshima University \\ Hiroshima, Japan, 739-8527 \\ Email: sozu@bsys.hiroshima-u.ac.jp \\ † Graduate School of Advanced Sciences of Matter, Hiroshima University \\ Hiroshima, Japan, 739-8527 \\ ${ }^{\ddagger}$ Graduate School of Engineering, Osaka University \\ Osaka, Japan, 565-0871
}

\begin{abstract}
Recently, the demand for odor processing apparatuses is increasing in the fragrance and food industries. In this paper, we propose a neural network model of the olfactory system as basis for biomimetic E-Nose. The model has two distinctive features. First, it converts the properties of odorant molecule to the neural activity pattern of living mice. Second, the olfactory "Attention" mechanism is introduced. The simulation results show that the model can predict the odor coding manner on the glomeruli by appropriately adjusting the parameters. Further, the tendency in accuracy rate of odor discrimination is consistent with that of living mice.
\end{abstract}

Index Terms-Olfactory System, Neural Network Model, Attention, Glomeruli activity pattern

\section{INTRODUCTION}

As considerable evidence has been reported that odors can affect human memory and emotion [1], the odor information and its evaluation method are becoming increasingly important, especially in the fragrance and food industries. Odor discrimination apparatus such as E-Nose is a reasonable solution for objective odor evaluation [2]. In addition, the E-nose would enable a moving robot to trace odors, which could be used as the robotic police dog [3]. However, odors are composed of combinations from tens of thousands of different odorant molecules, and form extremely high dimensional information. To reduce dimension of the information, the ability of most odor discriminating apparatuses were specialized for particular odor such as the E-Nose for banana ripeness developed by Llobet et al. [2]. Therefore, there are potential demands for higher performance E-nose. With such motivation, biomimetic approach, such as introducing model of the olfactory system, has begun to be utilized [4], [5], because animals discriminate a number of different odors in their daily life.

Although the ideal case is to make analysis on the olfactory system of humans, well-developed higher brain function would influence the natural odor sense, and make the problem more complex. Provided such limitations, this study pays attention to the olfactory system of mouse, because it is most investigated and necessary biological data is available.

The olfactory system must, first, convert the odor information into the activity pattern of neurons, then perform recognition based on the neuron-coded signals. Odors are perceived by receptor neurons distributed in the nasal and turned into specific activity patterns of glomeruli on the surface of the olfactory bulb. The main problem of this step is: how to convert numerous odorants into different activity patterns? The experimental data shown by Mori et al. [6] and Johnson et al. [7] suggest that the odorants sharing the similar chemical structure can stimulate the glomeruli at the similar location. The activity patterns are then transfered to the piriform cortex for odor recognition and discrimination. The system, then, have to classify the inputted activity patterns, and find the trade-off between robust classification and differentiation in similar odors. This task was studied at behavioral level by Okuhara et al. [9] where a series of odor discrimination experiments on mouse implied Attention mechanism exists in the olfactory system.

In this paper, an artificial neural network model is proposed based on the structure of the olfactory system of mice. Our model has two distinct features. First, odorant information is converted into activity patterns of neuron model similar to that of living mice. No such approach has been reported so far, because most of the previous studies have focused on the universal coding rules, but not on the actual activity patterns. Second, Attention mechanism is introduced to the model. Theses features enable the model to process odor information more animal-like. The ability of the model is compared to the results of odor discrimination experiments on mouse. To perform this comparison, a simple directional control part is built into the model.

\section{BIOLOGICAL INSIGHT}

\section{A. Olfactory system of mice}

This section briefs the basic structure of the olfactory system that are taken into consideration. Fig. 1 shows the 


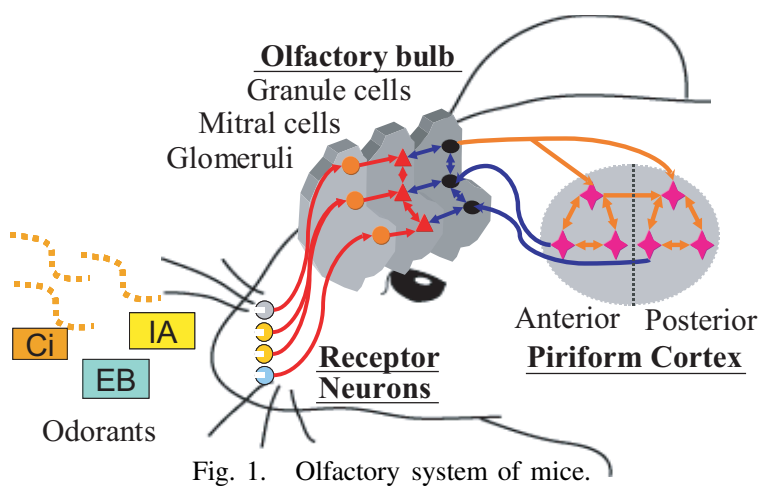

structure of the olfactory system of mice. The olfactory system is composed of three parts: receptor neurons, olfactory bulb, and piriform cortex. Receptor neurons that bind to specific odorants are distributed on the surface of the nasal chamber, expressing only one receptor gene from among a thousand different genes [8]. When odorant molecules bind to the receptor neurons, the neurons are activated and send signals to the olfactory bulb. The axons from the receptors that express the same gene terminate at the same point on the surface of the olfactory bulb [6]. The terminals of those axons form a small, round cluster called a glomerulus. A 2-D map of the glomeruli distribution could therefore be associated with the receptor genes as well as the odorants, and is thus called an odor map [6].

Besides the glomeruli, mitral cells and granule cells are the principal neurons in the olfactory bulb. The signals from the glomeruli are inputted to the mitral cells. The mitral cells are interconnected with each other through the excitatory and inhibitory synapses, which are mediated by the granule cells. In general, the olfactory bulb is considered to perform feature extraction [10].

The mitral cells transmit the signal to the pyramidal cells in the piriform cortex. The pyramidal cells transmit signals back to the granule cells in the olfactory bulb and indirectly inhibit the mitral cells. The piriform cortex is divided into the anterior piriform cortex (APC) and posterior piriform cortex (PPC); the division of their functions is slightly unclear. Generally, the piriform cortex is believed to be responsible for the identification of odors.

\section{B. Odor discrimination experiment}

Okuhara et al. has conducted a series of odor discrimination experiments on mice [9]. First, the mice are placed at the point $\mathrm{S}$, and trained to select a rewarded odor that emanates from either end E1 or E2 as shown in Fig. 2(a). The rewarded odor is composed of three types of odorants such as [IA, Ci, EB]. The trained mice are, then, required to discriminate the rewarded odor from other odors that share common odorants with the rewarded odor, such as [IA] or [IA, EB], and so on. Fig. 2(b) illustrates the results of the odor discrimination experiment on 10 mice. The results have shown that most of the mice have difficulty to discriminate [IA, EB] from [IA, Ci,

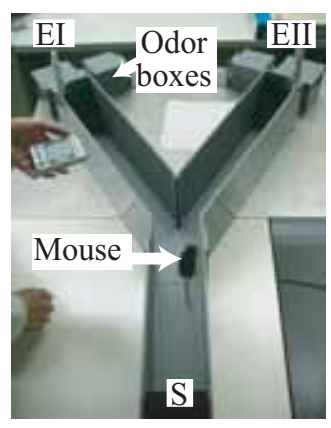

(a) Y-maze

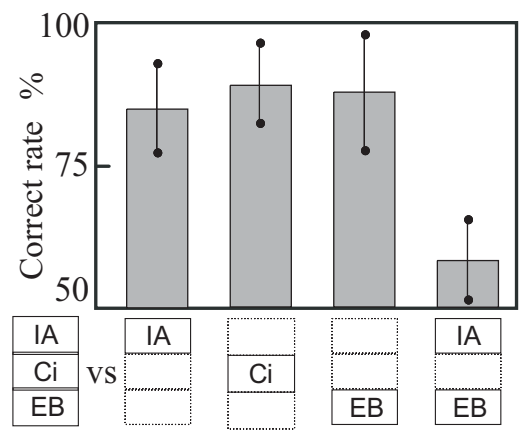

(b) Results of 24 trials
Fig. 2. Results of the odor discrimination experiment. (unpublished data) EB], and imply the mice provided attention to a combination of the odorants [IA] and [EB] when they were learning the odor [IA, Ci, EB]. Therefore, Attention mechanism similar to that of vision also exists in the olfactory system, and could largely contribute to characteristics of odor sensing. Although, the mice learned difference in those two odors, and improved the discrimination ability in the successive experiments, in this paper we would focus on the mechanism to establish Attention.

\section{Proposed Model OF THE OlFACTORY SYSTEM OF MICE}

A neural network model is proposed (Fig. 3) based on the structure of the olfactory system. The model decides direction to the reward based on the input odor just as the mice do in the odor discrimination experiments. In this section, the overview of the proposed model is described first, then the details of each part are given.

\section{A. General model structure}

The model consists of 4 parts: odor perception part, olfactory bulb part, piriform cortex part, and directional control part. The neuron population ${ }^{l} N$ composing layer $l$ are shown in Fig. 3.

The odor perception part is a feed-forward neural network of 3 layers, which are the preprocessing layer $(l=1)$, odorant layer $(l=2)$ and receptor layer $(l=3)$. This part converts the odorant properties into the activity pattern of the glomeruli based on the experiment data on mice [7].

The olfactory bulb part consists of the Glomeruli layer $(l=4)$, Mitral layer $(l=5)$, and Granule layer $(l=6)$. The population of neuron in these layers were determined based on the actual number of glomeruli distributed on the olfactory bulb [7].

The piriform cortex part consists of the $A P C$ layer $(l=7)$ and $P P C$ layer $(l=8)$, which correspond to anterior piriform cortex and posterior piriform cortex, respectively. It acts as associative memory and performs Attention together with the olfactory bulb. These mechanisms are introduced applying engineered approach, however, the connections between each layer in the olfactory bulb part and piriform cortex 


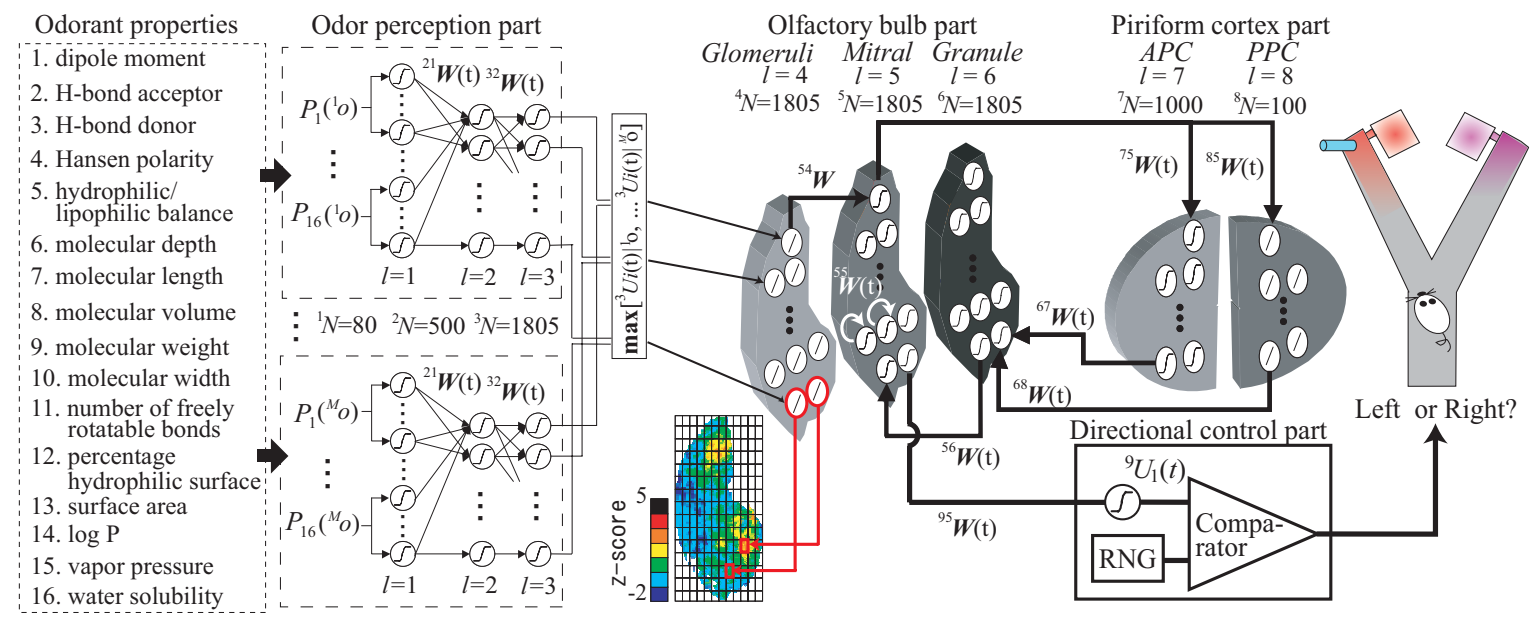

Fig. 3. Structure of the proposed model. The activity pattern of glomeruli is cited from the literature [7]

part are set up based on the structure of the olfactory system described in Section II-A. Note that the interconnections in each layer are not included in the model for simplification, thus the memory searching ability is omitted.

The directional control part, which determines the direction leading to the rewarded odor, is introduced to simulate the odor discrimination experiment described in Section II-B. This part is composed of a single neuron, random number generator (RNG), and a comparator.

Next subsection details each part of the model.

\section{B. Details of the model}

The perception part takes the odorant properties as the input from the preprocessing layer. Because approximately 400,000 kinds of odorants exist, forming extremely high dimensional information, it is impossible to input the odorant information to the model in a binary coding manner. Thus, the properties of each odorant are broken down into 16 numeric properties $(P=16)$ listed in the left side of Fig. 3. These properties have been listed by Johnson et al. [11] along with the corresponding activity patterns.

In order to process the properties with different units and orders, the preprocessing layer converts the value of the properties into the activated neuron numbers for normalization. This method is introduced based on the concept of population coding [12]. For this purpose, the neurons in the preprocessing layer are divided into 16 groups, and each group receives a different kind of odorant property. Therefore, the input to the neurons in the preprocessing layer is given by the following equation:

${ }^{1} u_{s}(t)=P_{i}\left({ }^{m} o\right), \quad(s=(i-1) K+k, k=1,2 \ldots K)$,

where ${ }^{1} u_{s}(t)$ is the input to the $s^{\text {th }}$ neuron in the preprocessing layer $(l=1)$ at time step $t ;{ }^{m} O$, the $m^{\text {th }}$ odorant in odor $O ; P_{i}$, the $i^{\text {th }}$ numeric property of the odorant ${ }^{m} O$; and $\mathrm{K}$, the maximum number of neurons responsible for property $P_{i}$. The inputs are transferred into the activity of the neurons by the following sigmoid function:

$$
{ }^{1} U_{s}(t)=\frac{1}{1+\exp \left\{-{ }^{1} \epsilon_{s}\left({ }^{1} u_{s}(t)-{ }^{1} \theta_{s}\right)\right\}} .
$$

The outputs of sigmoid neurons in other layers are also calculated by the above equation. The threshold ${ }^{1} \theta_{s}$ and the gradient ${ }^{1} \epsilon_{s}$ of the sigmoid function are determined according to the corresponding property by the following equation:

$$
\begin{aligned}
{ }^{1} \theta_{s} & =k \frac{\left(P_{i, \max }-P_{i, \min }\right)}{K}, \\
{ }^{1} \epsilon_{s} & =C_{s} \frac{\left(P_{i, \max }-P_{i, \min }\right)}{K},
\end{aligned}
$$

where $P_{i, \max }$ and $P_{i, \min }$ are the maximum and minimum values of the property $P_{i}$ in an odorant data set, and $C_{s}$ is a constant.

The output of the preprocessing layer $(l=1)$ is inputted to the odorant layer $(l=2)$ through a connective weight matrix ${ }^{1} \mathbf{W}(t)$. The input to the odorant layer is given by the following equation:

$$
{ }^{2} u_{n}(t)=\sum_{s}{ }^{21} w_{n s}(t){ }^{1} U_{s}(t),
$$

where ${ }^{2} u_{n}(t)$ is the input to the $n^{\text {th }}$ neuron in the odorant layer, and ${ }^{2} 1 w_{n s}(t)$ is the connective weight between neuron units $n$ and $s$, which is an element in the connective weight matrix ${ }^{21} \mathbf{W}(t)$. The odorant layer reconstructs the odorant that is divided into 16 numeric properties in the preprocessing layer. This layer is introduced to for avoiding the loss of information caused by compressing the information from the approximately 400,000 odorant dimensions into the 16 property dimensions.

The output of the odorant layer is inputted to the receptor layer $(l=3)$ through ${ }^{32} \mathbf{W}(t)$. The input of the receptor layer is given by the following equation:

$$
{ }^{3} u_{r}(t)=\sum_{m}{ }^{2} w_{r n}(t){ }^{2} U_{n}(t),
$$


This layer acts as the receptor that responds to the bound odorants. The output of the neuron in this layer represents the average response of a receptor cell expressing the same receptor gene.

The output of the receptor layer $(l=3)$ is passed to the glomeruli layer $(l=4)$. According to Lin et al. [13], the activity of the glomeruli evoked by odor $O$ can be binary addition of the activity patterns evoked by its odrant components. Thus, the input and output of the Glomeruli layer is determined by the following equation:

$$
{ }^{4} u_{e}(t)=\max \left[{ }^{3} U_{r}(t)\left|{ }_{o_{o}} \ldots{ }^{3} U_{r}(t)\right|{ }_{M_{o}}\right]
$$

The output ${ }^{4} U_{e}(t)$, which is calculated by Equation (2), is an element in the activity pattern vector ${ }^{4} U(t)$. Each element in the activity pattern vector corresponds to a divided lattice of the activity patterns provided by Johnson et al. [7], as shown in Fig. 3.

The output of the Glomeruli layer is input to the Mitral layer. The Mitral layer forms 2 closed loop with $A P C$ and $P P C$ layer through Granule layer as shown in Fig. 3. The input and output of the neurons in these layer are given by the following equations:

$$
\begin{aligned}
{ }^{5} u_{b}(t) & =\sum_{e}{ }^{54} W_{b e}(t)^{1} U_{e}(t)+\sum_{d}{ }^{55} W_{b d}(t)^{2} U_{d}(t) \\
& +\sum_{m}{ }^{56} W_{b g}(t)^{3} U_{g}(t) \\
{ }^{6} u_{g}(t) & =\sum_{z}{ }^{67} W_{g z}(t)^{7} U_{z}(t)+\sum_{x}{ }^{68} W_{g x}(t)^{8} U_{x}(t) \\
{ }^{7} u_{z}(t) & =\sum_{b}{ }^{75} W_{z b}(t)^{5} U_{b}(t)
\end{aligned}
$$

The outputs of the neurons are given by Equation (2) with substitution of the layer number. However, the output of the neurons in the $P P C$ layer is proportional to its input considering the restrictions imposed by the learning algorithm described in the next section.

The output of the Mitral layer is then input to the neuron in the directional control part. Its input and output are calculated using the following equation,

$$
{ }^{9} u_{1}(t)=\sum_{b}{ }^{95} W_{1 b}(t){ }^{5} U_{b}(t),
$$

The output ${ }^{9} U_{1}(t)$ is computed by Equation (2), then compared with the output of RNG, which generates uniform random numbers on $[0,1]$, to decide the direction of rewarded odor.

\section{The learning phase}

The learning algorithm for the neural network model described in the previous section consists of 2 steps. In the $1^{\text {st }}$ step, the actual activity patterns of glomeruli are utilized to train the proposed model so that the Glomeruli layer can produce activity pattern corresponding to the input odors.
Then, in the $2^{\text {st }}$ step, the model is trained to extract feature in the activity patterns of the Glomeruli layer based on the odor discrimination experiment. The details of each step are described bellow.

1) The $1^{\text {st }}$ step of the learning phase: In the $1^{\text {st }}$ step, the connective weights ${ }^{21} \mathbf{W}(t)$ and ${ }^{32} \mathbf{W}(t)$ are adjusted to produce activity patterns of Glomeruli layer corresponding to the inputted odorants' properties given by the training set. The connective weights are adjusted to minimize the mean square error (MSE) between the output of the glomeruli layer and activity measured from actual glomeruli. For implementation of weight adjustment, the RPROP algorithm proposed by Riedmiller et al. [14] is utilized.

2) The $2^{\text {nd }}$ step of the learning phase: In the $2^{\text {nd }}$ step, the connective weights in the olfactory bulb and piriform cortex are modulated based on the algorithm that we have proposed [15]. This learning simulation is performed using the connective weights that are adjusted in the previous simulation. Because most computational functions of the olfactory system have not been revealed clearly, in this paper, the signal transduction or the modulation of connective weights between the neurons are hypothesized based on the odor discrimination experiment described in section II-B [9]. We assume that the olfactory system extracts the most activated regions in the glomeruli. With regard to these assumptions, we propose a learning algorithm that consists of the following 3 steps.

In the $1^{\text {st }}$ step, the connective weights ${ }^{75} \mathbf{W}(t)$ and ${ }^{67} \mathbf{W}(t)$ are modulated to extract activated parts from the background activity of the neuron. The output of the $b^{\text {th }}$ neuron in the Mitral layer to an arbitrary odor A is assumed to be $\left.{ }^{5} U_{b}(t)\right|_{{ }_{A}}$; the background activity, $\left.{ }^{5} U_{b}(t)\right|_{\text {back. Then, }}$ assuming that the odors are identified in the $A P C$ layer, the outputs of the $z^{\text {th }}$ neuron in the $A P C$ layer are preliminarily determined to be $\left.{ }^{7} U_{z}(t)\right|_{{ }_{A}} O$ and $\left.{ }^{7} U_{z}(t)\right|_{\text {back }}$ for the odor A and background activity, respectively. Provided these assumption, the connective weights ${ }^{75} W(t)$ and ${ }^{67} W(t)$ are modulated by the following equation:

$$
\begin{aligned}
& { }^{75} W_{z b}(t+1)=\alpha^{75} W_{z b}(t)+\left.\left.\beta^{5} U_{b}(t)\right|_{A} O^{7} U_{z}(t)\right|_{A} O,(12) \\
& { }^{67} W_{g z}(t+1)=\alpha^{67} W_{g z}(t)+\beta^{7} U_{z}(t)\left|{ }_{A}{ }^{5} U_{b}(t)\right|_{\text {back }}(13)
\end{aligned}
$$

where $\alpha$ denote the forgetting term; $\beta$, the learning rate. When odor $\mathrm{A}$ is input, through the connective weights ${ }^{75} W(t)$ and ${ }^{67} W(t)$, the background activity of the Mitral layer is produced on the Granule layer. Then, the outputs of the Granule layer inhibit the neurons in the Mitral layer through ${ }^{56} W_{b g}=-\delta_{b g}$. Consequently, the background activity on the Mitral can be subtracted by this step.

In the $2^{\text {nd }}$ step, the most activated neurons are extracted. After the learning step 2, it is considered that the neurons of the Mitral layer with the strongest outputs could be the most effective feature of the odor A. In the proposed model, we assume that the connective weights between the Mitral layer 
and the $P P C$ layer form a competitive system. Thus, the corresponding connective weights are determined according to the competitive system proposed by Amari et al. [16]; these connective weights include self-feedback connective weights ${ }^{55} \mathbf{W}(t)$ in the Mitral layer, feed forward connective weights ${ }^{85} \mathbf{W}(t)$ from the Mitral layer to the $P P C$ layer, and the feedback connective weights ${ }^{68} \mathbf{W}(t)$ from the $P P C$ layer to the Granule layer.

Finally, in the $3^{\text {rd }}$ step, the activity pattern of the Mitral layer after the $2^{\text {nd }}$ step is memorized by adjusting the connective weights ${ }^{95} \mathbf{W}(t)$; the weights are determined by the following equation:

$$
{ }^{95} W_{1 b}(t)={ }^{5} U_{b}(t) \mid{ }_{A} O .
$$

\section{Directional control}

In the directional control simulation, an arbitrary odor B is inputted to the model. The input to the directional control part can be calculated by using Equation (11) as follows:

$$
\begin{aligned}
\left.{ }^{9} u_{(}(t)\right|_{{ }_{B}} & =\left.\sum_{b}{ }^{95} W_{1 b}(t)^{5} U_{b}(t)\right|_{B O} \\
& =\sum_{b}{ }^{5} U_{b}(t)\left|{ }_{A} O{ }^{5} U_{b}(t)\right|_{B} O .
\end{aligned}
$$

Accordingly, calculating the input is equivalent to calculating the correlation between the current output $\left.{ }^{5} U_{b}(t)\right|_{B O}$ and the memorized output $\left.{ }^{5} U_{b}(t)\right|_{A} O$ of the Mitral layer. Now, assuming the proposed model is sniffing an odor sent from right arm of the Y-maze, if the output of the neuron is larger than that of RNG, then the directional control part would choose the right arm, otherwise it choose the left arm. The RNG may represents the randomness in mices' behavior as they scarcely make $100 \%$ of accuracy rate, or choose wrong end on purpose to confirm their given task.

\section{Simulation}

This section describe the simulations that are performed based on the algorithm described in the previous section.

\section{A. The $1^{\text {st }}$ step of the simulation}

First, 70 odorants data are randomly selected from the 365 odorants provided by Johnson et al. [11] to form the first part of the training set (training set I). Then, several odorants are chosen arbitrary for the second part of the training data (training set II). The properties of the odorants in the training set are input to the model, and the connective weights are adjusted by the RPROP algorithm.

Fig. 4 shows the outputs of the model after the training is completed. In Fig. 4, the molecules in the uppermost row are the inputted odorants, which are followed by the actual activity patterns [7], the output of the model, and the graph of MSEs. The odorants with a gray background are included in the training set, while those with no background color are not. From this figure, we can observe that the MSE for the training

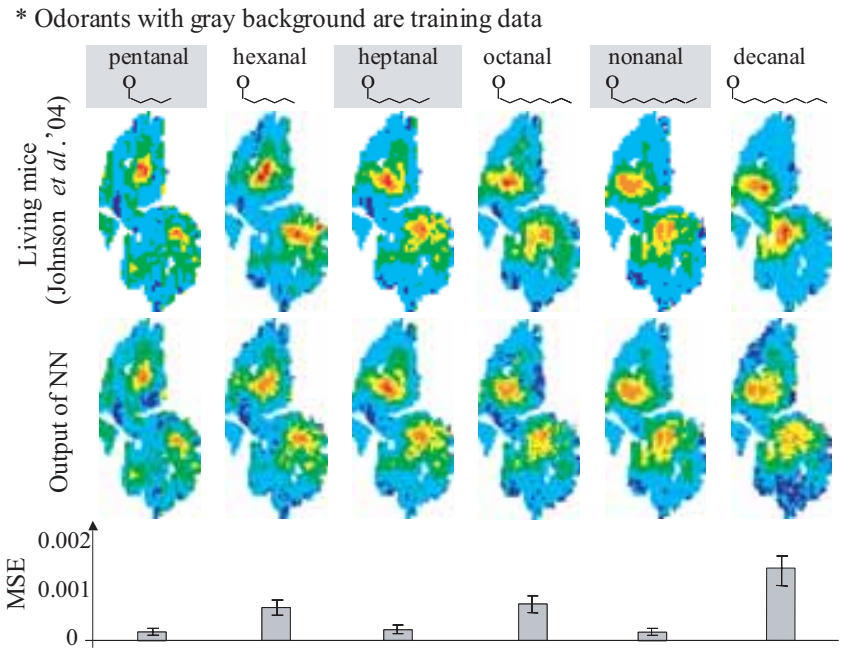

Fig. 4. Simulation results of aldehydes.

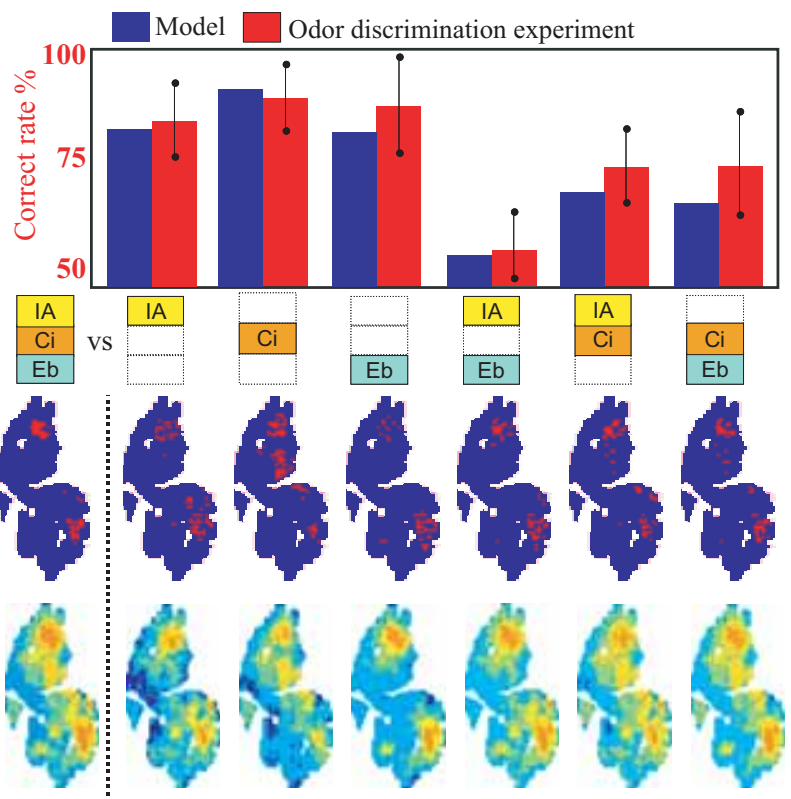

Fig. 5. Comparison between the results of the behavior experiments.

set is below 0.002 , while that for the untrained odors ranges from about 0.007 to 0.015 . The reason for decanal recording the highest MSE is that, unlike in the case of hexanal and octanal, an odorant with one more carbon is not included in the training set. Overall, it can be observed that the model could interpolate the tendency in the continuous changing of activity patterns along with the carbon number.

\section{B. The $2^{\text {nd }}$ step of the simulation}

In the learning phase, an odor [IA, Ci, EB], which stands for an odorant mixture composed of isoamyl acetate, citral, and ethyl butyrate, is input to the proposed model; The connective weights then are adjusted according to the learning algorithm described in Section III-C2. The initial values of the connective weights are determined by the uniform random values ranged between $-10^{-5}$ and $10^{-5}$. 
After the learning phase, 6 different odors [IA], [EB], [Ci], [IA, EB], [IA, Ci], and [Ci, EB] are input to the model, and 1000 trials were attemptted for each odor. Note that, the connective weights are fixed at the values determined in the $1^{\text {st }}$ step. Then, the decisions made by the directional control part are compared with the correct rates in odor discrimination experiments on the mice. The accuracy rate of the directional control part is defined as the ratio of choosing rewarded odor [IA, Ci,EB] to the total trial number.

The outputs of the Glomeruli layer to each odor are shown in the lowest row in Fig. 5. The outputs of the Mitral layer after the $2^{\text {nd }}$ learning phase are shown in the middle row in Fig. 5. The accuracy rate of the decisions made by the directional control part are plotted at the top of Fig. 5. Further, the correct rates obtained from the odor discrimination experiment on the mice are plotted beside the accuracy rate of the directional control part.

Comparing the activity patterns of the Glomruli layer to that of the Mitral layer, it can be observed that the activated region becomes narrow, but its activity becomes stronger. Therefore, the model extracted the most activated region in the Glomruli layer. As described in Section II-A, the directional control part make decision based on the pattern on the Mitral layer, thus, the similar extracted pattern with that of rewarded odor would lower the accuracy rate. This tendency is illustrated in Fig. 5, from which we can observe that the accuracy rates of the experiment and the model are highly correlated to each other. From this result, we can conclude that, up to an extent, the feature extraction property of the model is consistent with Attention mechanism of the living mice.

\section{CONCLUSION}

In this paper, we proposed a neural network model of the olfactory system of mice. Utilizing the model, we tried to predict the activity pattern in glomeruli evoked by odorants. The simulation results showed that the model was capable of predicting the activity patterns of untrained odors that had different carbon numbers with the trained odors, and showed consistency with those of the odor discrimination experiments on mice, which indicated that the model has an ability similar to Attention. Although we only discussed about odor discrimination characteristics affected by Attention in this paper, by adjusting the parameters in the model, relearning ability can also be introduced by adjusting the connective weights.

However, odors in nature are composed of odorants in different concentrations, which is not accounted for in the proposed model. Therefore, in the next step, the odor coding manner for different concentration have to be studied. In addition, as we have only performed Attention simulations on odorants [IA], [Ci], and [EB] with limitation of experiment data, we plan to conduct further odor discrimination experiments on mice by using other types of odorants. Further, to simulate more animal-like perception, we would like to introduce interconnection between glomeruli and consider the signals from other parts of the olfactory system.

Acknowledgments.: This work is partially supported by the 21st Century COE Program of JSPS (Japan Society for the Promotion of Science) on Hyper Human Technology toward the 21st Century Industrial Revolution.

The authors would like to thank Dr. Keisuke Okuhara for his biological advice and Dr. Michiyo Suzuki and Dr. Akira Hirano for their editing skills and continuing support.

\section{REFERENCES}

[1] R.S. Herz and T. Engen, "Odor memory: review and analysis," Psychonomic Bulletin and Review, Vol. 3, No. 3, pp. 300-313, 1996.

[2] E. Llobet, E.L. Hines, J.W. Gardner, and S. Franco, "Non-destructive banana ripeness determination using a neural network-based electronic nose," Measurement Science and Technology, Vol.10, pp. 538-548, 1999.

[3] A.W. Teo, H.K. Garg, and S.Puthusserypady, "Detection of humans buried in rubble: an electronic nose to detect human body odor," Proceedings of the Second Joint EMBS/BMES Conference, Vol. 3, pp. 1811-1812, 2002.

[4] R. Gutierrez-Osuna, "Pattern Analysis for Machine Olfaction: A Review," IEEE Sensors Journal, Vol. 2, No. 3, pp. 189-202, 2002.

[5] B. Raman, T.Yamanaka, and R. Gutierrez-Osuna, "Contrast enhancement of gas sensor array patterns with a neurodynamics model of the olfactory bulb," Sensors and Actuators: B Chemical, Vol. 119, No. 2, pp.547-555, 2006.

[6] K. Mori and Y. Yoshihara "Molecular recognition and olfactory processing in the mammalian olfactory system," Progress in Neurobiology, Vol. 45, pp. 585-619, 1995.

[7] B.A. Johnson and M. Leon "Modular glomerular representations of odorants in the rat olfactory bulb and the effects of stimulus concentration," The Journal of Comparative Neurology, Vol. 422, pp. 496-509.

[8] L. Buck and R. Axel "A novel multigene family may encode odorant receptors: a molecular basis for odor recognition," Cell, Vol. 65, pp.175187, 1991

[9] K. Okuhara and T. Nakamura "Explore algorithms in olfactory system of mice," Software Biology, Vol.3, pp.20-25, 2005. (in Japanese)

[10] O. Hoshino, Y. Kashimori, and T. Kambara, "Feature extraction from mixed odor stimuli based on spatio-temporalrepresentation of odors in olfactory bulb," International Conference on Neural Networks, vol. 1, pp. 344-349, 1997.

[11] M. Leon and B.A. Johnson, "Glomerular Response Archive," http://leonserver.bio.uci.edu/.

[12] A.P. Georgopoulos, A.B. Schwartz, R.E. Kettner, "Neuronal population coding of movement direction," Science, Vol. 233, Issue. 4771, pp. 1416-1419, 1986.

[13] D.Y. Lin, S.D. Shea, L.C. Katz, "Representation of natural stimuli in the rodent main olfactory bulb," Neuron, Vol.50, No. 6, pp. 816-818, 2006.

[14] M. Riedmiller and H. Braun, "A direct adaptive method for faster backpropagation learning: The RPROP algorithm," Proceedings of the IEEE International Conference on Neural Networks San Francisco, pp. 586-591, 1993.

[15] Z. Soh, T. Tsuji, N. Takiguchi, H. Ohtake "A neural network model of the olfactory system of mice Simulated the tendency of attention behavior," The 13th International Symposium on Artificial Life and Robotics, pp. 537-542, 2008.

[16] S. Amari, M.A. Arbib, "Computation and cooperation in neural nets," Systems Neuroscience, Academic Press, pp. 67-117, 1977. 